\title{
De la (curiosísima) relación entre la Medicina Basada en la Evidencia y la práctica psiquiátrica en nuestro entorno.
}

\author{
On the (quaint) relationship between Evidence-Based \\ Medicine and Psychiatric Practice in our environment.
}

\begin{abstract}
RESUMEN: El modelo psiquiátrico más extendido y aceptado en la actualidad es el de la llamada psiquiatría biológica, siendo una de sus herramientas predilectas la medicina basada en la evidencia (MBE). Sin dejar a un lado las críticas hechas al modelo y las deficiencias de tal herramienta, nos hemos querido plantear también si realmente esta misma psiquiatría biológica y sus seguidores aceptan las conclusiones obtenidas a partir de la MBE, comentando diferentes trabajos de resultados que hemos considerado interesantes. Terminamos con unas opiniones personales sobre la cuestión de, si no es la MBE, qué o quién aporta la energía para la práctica de la psiquiatría biológica tal como se realiza en nuestro entorno. PALABRAS CLAVE: Medicina Basada en la Evidencia. Psiquiatría Biológica. Positivismo. Empirismo.
\end{abstract}

\begin{abstract}
Currently, most extended and accepted psychiatric model is the so-called biological psychiatry, being Evidence-Based Medicine one of its most favourite tools. Without leaving aside criticism made to the model and those tool's flaws, we have wanted to set out if biological psychiatry itself and its supporters do really accept conclusions drafted by EBM, discussing some work's results, which we have considered interesting. We finish up with some personal opinions about the matter of, if it is not EBM, what or who is supplying power to the practice of biological psychiatry as it is carried out in our environment.

KEY WORDS: Evidence-Based Medicine. Biological Psychiatry. Positivism. Empiricism.
\end{abstract}

Donde se cuenta (aunque no se comparta) el dominio del paradigma médico en nuestra disciplina a través de la llamada psiquiatría biológica

Desde nuestras diferentes profesiones y aproximaciones al campo de la salud mental, creemos poco discutible la afirmación de que es la llamada psiquiatría biológica la visión predominante en nuestra disciplina en la actualidad. Por supuesto, existen excepciones a este dominio con tintes de exclusividad, pero son la mayoría de las veces eso: excepciones. El paradigma médico, junto al cognitivo-conductual, parten de postulados positivistas y empiristas en busca de un reconocimiento de verdad y saber que creen encontrar en ese ambicionado y proclamado estatus de ciencia. Otros acercamientos a la locura, al malestar o al deseo como el psicoanálisis o las terapias humanistas y existenciales son arrumbadas bajo el oprobio de no ser científicos.

En su manual Introducción a la psicopatología y la psiquiatría, Julio Vallejo (1), hablando de las tendencias doctrinales en la psiquiatría actual, concre- 
tamente de las órgano-mecanicistas, recoge en un par de párrafos lo que podría considerarse la posición más o menos explícita de gran parte de los psiquiatras de nuestro país y otros de nuestro entorno: "La realidad es que, en las últimas décadas, la psiquiatría se ha decantado nuevamente hacia el polo de la medicina y defiende para algunos trastornos (esquizofrenia, trastornos afectivos, estados obsesivos, crisis de angustia) etiologías claramente biológicas. [...] La psiquiatría es una rama de la medicina [y] debería utilizar metodologías científicas modernas y basar su práctica en el conocimiento científico. [...] El foco de atención de los médicos psiquiatras debería centrarse particularmente en los aspectos biológicos de la enfermedad" (2). Entendemos que estas palabras no son la opinión literal del autor sino la descripción de un determinado enfoque teórico. Vallejo no deja de tener en cuenta en distintas partes de su obra otras posiciones teóricas y prácticas en el abordaje de la patología mental pero, sin embargo, creemos que estas breves líneas dibujan claramente la posición de lo que podríamos llamar la psiquiatría oficial y académica. El párrafo recogido literalmente no tiene desperdicio: habla de etiologías claramente biológicas, sin que esa claridad sea de tal calibre para que todos los autores las reconozcan, situando posiblemente correlaciones estadísticas al nivel de explicaciones etiológicas, sin quedar a salvo de hipotéticas confusiones entre causas y efectos del trastorno e incluso tal vez sin distinguir si las alteraciones biológicas halladas no son a su vez provocadas por los tratamientos empleados. Por otro lado, la recomendación a los médicos psiquiatras de basar su práctica en el conocimiento científico nos lleva directamente a la cuestión de la llamada, en una mala traducción, medicina basada en la evidencia (MBE), sobre la cual volveremos luego con amplitud. Un último apunte sólo para señalar el interesante pleonasmo de médico psiquiatra, tan frecuente por otra parte. No hacen falta especiales dotes como psicoanalistas, de las que por otra parte carecemos, para interpretar el posible malestar o temor del psiquiatra a no ser reconocido como médico. Como nos dijo un compañero una vez, nunca se oyó a nadie presentarse como médico traumatólogo.

Siguiendo a Luque y Villagrán en su brillante Psicopatología descriptiva: nuevas tendencias (3), el paradigma médico se basa en el modelo de enfermedad anatomo-clínico que aparece en el siglo XIX y que se extiende hasta la actualidad. Como señala Berrios (4), citado en esa obra, en el siglo XIX la medicina, en su aspecto de lenguaje para describir y/o explicar la enfermedad, se reinterpretó conforme a la filosofía científica de la época y las presiones éticas se centraron en la necesidad de someter las enfermedades al método positivista, es decir, la credibilidad de los hallazgos médicos dejó de estar basada en la tradición o la autoridad y pasó a fundamentarse en la capacidad de predicción y en la validación experimental. Para este paradigma médico, la psicopatología es una forma de descripción médica, los trastornos mentales constituyen enfermedades y la 
ORIGINALES Y REVISIONES

conceptualización y el estudio de las enfermedades mentales deben ser fundamentalmente biológicos. La conducta anómala sería consecuencia de los cambios físicos y químicos que ocurren generalmente en el cerebro.

Según Maher (5) el paradigma médico supone, entre otras cosas, que la persona que muestra determinadas alteraciones respecto a los rasgos considerados normales en una población está enferma (lo que hace depender la definición de enfermedad de algo tan poco científico como los rasgos considerados normales en una población) y que la enfermedad es consecuencia de una etiología demostrable, o que se supone se evidenciará en el futuro, la cual es, en principio y por definición, biológica u orgánica (consideramos también interesante esa proyección hacia el futuro del hallazgo de las causas, aunque esto de confiar en anunciadas venidas futuras nos parece que tiene más resonancias de fe religiosa que de ciencia positiva...).

Siguiendo a Luque y Villagrán, la investigación psiquiátrica actual está dominada por una posición epistemológica empirista, que surge de la tradición filosófica del empirismo inglés (Locke, Berkeley, Hume), el sensacionalismo francés (La Mettrie), el positivismo de Comte y la filosofía analítica del Círculo de Viena (Carnap, Neurath, Schlick). Para el psiquiatra empirista, el conocimiento surge fundamentalmente de la observación, por lo que se ha desarrollado una metodología de investigación con el fin de controlar los sesgos que puedan aparecer. Los presupuestos epistemológicos de la psiquiatría empirista se hacen patentes, por ejemplo, en el uso de escalas de evaluación, entrevistas estructuradas, definiciones y criterios operativos de síntomas y trastornos, sistemas clasificatorios como los DSM, el empleo de la estadística, etc. (podríamos decir que al paciente se le pregunta mucho pero se le escucha poco). La mayoría de los psiquiatras en formación están familiarizados con esta actitud científica pero sin conocer el marco filosófico del que parte ni las limitaciones de éste, principalmente en relación con los presupuestos ontológicos (anti-realistas) que acompañan a esta posición epistemológica empirista. No nos detendremos en este punto por exceder el propósito de este artículo, aunque recomendamos la lectura de la obra citada de Luque y Villagrán. Como señalan también estos autores, gran parte de la ciencia psiquiátrica actual se presenta como biológica pero en realidad asume un concepto reduccionista de lo biológico en lo fisicoquímico, con lo que se prescinde del nivel de descripción propiamente biológico, es decir, el que trata de los procesos que un organismo lleva a cabo en relación con una serie de medios diferentes a los que intenta adaptarse mediante su representación, la regulación de su funcionamiento y la elaboración de respuestas adaptativas.

Establecidos estos apuntes básicos sobre las características del paradigma médico en psiquiatría, nos detendremos ahora en una de sus herramientas predilectas: la MBE. Diremos que la psiquiatría biológica dominante, desde 
sus fundamentos eminentemente empiristas y su doctrina positivista encuentra el combustible perfecto para su viaje en los principios y prácticas de la MBE. Lo primero a destacar, como revisa Lázaro (6) es el por suerte cada vez más conocido error de traducción. El término original en inglés es "evidence based medicine" pero, como señala Fernando A. Navarro (7) en su Diccionario crítico de dudas inglés-español de medicina, en español "evidencia" significa "certeza clara, manifiesta y tan perceptible de una cosa, que nadie puede racionalmente dudar de ella". Por otra parte, el término inglés "evidence" suele emplearse para indicar un grado mucho menor de certeza y equivale a lo que sería en nuestro idioma: "indicios, signos, datos, pruebas, hechos indicativos o datos sugestivos". Como indica Lázaro, esta confusión entre "evidence" y "evidencia" esconde un muy peligroso error conceptual, sugiriendo certeza donde sólo hay probabilidad, pruebas, indicios. Error no exento de consecuencias a nivel teórico y práctico, reforzando la seguridad de muchos médicos psiquiatras de estar trabajando, no sólo en un campo plenamente científico y respetable, sino además con abundancia de estudios, patrocinados por la industria farmacéutica, a su disposición, con resultados aparentemente evidentes para insistir en la prescripción de determinados psicofármacos de precios considerablemente elevados. ¿Sería otro nuestro comportamiento si las conclusiones de esos estudios no nos vinieran presentadas como basadas en la evidencia, sino como, traduciendo correctamente, basadas en indicios?

Siguiendo a Desviat (8), la MBE pretende ser el camino hacia la verdad científica, presentándose como un nuevo paradigma científico, es decir, una matriz teórica que proporciona un marco conceptual de los fenómenos estudiados en la disciplina, capaz por tanto de sustituir los paradigmas previos en psiquiatría. Según este planteamiento, deudor del pensamiento de Kuhn (9), se habría producido un cambio en la percepción de la psiquiatría, una nueva forma de entender su campo de actuación, métodos y prácticas. La MBE supondría la superación de la incertidumbre en la toma de decisiones clínicas y de planificación y gestión. Haciendo un poco de historia, en 1988 Laupacis y colaboradores (10) señalaron que los clínicos no tenían un patrón de medida con el que comparar los riesgos y beneficios de los diferentes abordajes terapéuticos. Para que la medicina, y, en lo que nos interesa, la psiquiatría, pudiese ser científica, debería someterse a un proceso de verificación empírica, basado en pruebas. En 1992 se publica en la revista $J A M A$ el artículo fundacional de la $\mathrm{MBE}$, firmado por el autodenominado Evidence-Based Medicine Working Group (11). El grupo de trabajo dirigido por Sackett propugnaba un cambio de paradigma basado en una serie de axiomas:

- La experiencia clínica y la intuición en ocasiones pueden resultar engañosas.

- El estudio y comprensión de los mecanismos básicos de la enfermedad constituyen guías necesarias pero insuficientes en la práctica clínica, pudiendo 
ORIGINALES Y REVISIONES

llevar a predicciones incorrectas. El conocimiento psicopatológico y la experiencia clínica no son suficientes para establecer juicios como el diagnóstico, el pronóstico y la eficacia de los tratamientos; dan lugar a una medicina basada en la opinión que puede conducir a predicciones inexactas.

- Es preciso buscar la mejor evidencia disponible a través de búsquedas en la literatura científica.

Como señala Sackett (12), el ensayo clínico aleatorizado, especialmente la revisión sistemática de varios ensayos clínicos aleatorizados o metaanálisis, es el "patrón oro" para juzgar si un tratamiento causa o no beneficio o daño. Como afirma Desviat, la decisión clínica se convierte en el resultado de un proceso supuestamente objetivo y reproducible. Frente al llamado "ojo clínico" y las conjeturas y suposiciones basadas en la experiencia e imposibles de validar según criterios de ciencia natural, la MBE ofrece verificaciones empíricas, pruebas (aunque mal traducidas como evidencias, eso sí).

Un tratado de psiquiatría de uso relativamente frecuente en nuestro país, como es el de Vallejo Ruiloba y Leal Cercós, dedica un capítulo completo en su sección de "Generalidades" a lo que llama, empleando en este caso una traducción correcta del término anglosajón, Psiquiatría basada en pruebas (13). Se afirma en este capítulo: "Vaya por delante, en definitiva, la convicción de que las decisiones clínicas médicas -y entre ellas obviamente las psiquiátricas, éstas a partir de una psiquiatría basada en pruebas (PBP)- deben fundamentarse en pruebas científicas demostradas. Se trata de un hecho incuestionable y ante el que no cabe escatimar esfuerzos". La declaración de principios es, ésta sí, evidente. Más tarde podremos detenernos en comprobar la aplicación práctica de estos principios que llevamos a cabo los distintos profesionales de la salud mental. Volviendo al texto ya citado de Desviat, no es oro todo lo que reluce, y los metaanálisis también tienen sus limitaciones. Entre otras, señalaremos que el poder de generalización de un estudio o validez externa puede estar amenazado, si los profesionales que participan o la institución donde se lleva a cabo no son representativos, o bien tienen conflictos de intereses en el asunto investigado, o bien los pacientes pueden ser atípicos por el procedimiento de selección. Por otra parte, sólo algunos de los estudios que se realizan son publicados, preferentemente los que tienen resultados positivos y escritos en inglés.

Como Desviat comenta, la MBE reflejaría un intento ingenuo de elevar unas técnicas y una metodología de búsqueda e investigación al nivel de un nuevo paradigma, revolucionario por definición en el sentido de Kuhn, capaz supuestamente de dar cuenta de toda la realidad de la práctica psiquiátrica. Pero una colección de hechos no basta para constituir una ciencia, ni siquiera una ciencia aplicada. Un paradigma es un conjunto de teorías y algunas aplicaciones ejemplares capaces de dar una explicación coherente de un determinado campo del saber. 
La cuestión es que la MBE y sus seguidores no parecen conformarse con ser unas técnicas y una metodología, sino que, como comentamos previamente, quieren ir algunos pasos más allá, arrogándose la condición de paradigma capaz de resolver todas las viejas discusiones de la psiquiatría. La MBE coincide y se relaciona con una época de retroceso de la teoría psiquiátrica, del estudio psicopatológico y de los programas de investigación, más allá de la genética y la psicofarmacología. En las últimas décadas ha sido clara la vuelta al reduccionismo biológico o, más bien, fisicoquímico. Para algunos de sus adalides $(14,15)$ el futuro de la psiquiatría es convertirse en una "neurociencia clínica". Otros se preguntan si la psiquiatría no debería volverse una subdisciplina de la neurología. Este reduccionismo se refleja en las formas de entender la organización de la asistencia y el cuidado a los problemas de la salud mental: se producen cambios en los sistemas de pago por seguro en EEUU que exigen precisión diagnóstica, la financiación de las investigaciones, en gran parte en manos de la empresa privada, comienza a privilegiar los estudios biomédicos, con el pretexto de una mayor necesidad de objetivación, se reformula la nosología psiquiátrica a partir de la publicación del DSM-III en 1980, donde se hace ya patente la retirada de la psicopatología frente a criterios de agrupación por síntomas y evolución.

Esta nueva, y triunfante hasta nuestros días, nosología pretende ser "científica", con sus categorías basadas en supuestos datos objetivos, claros, únicos, universales y, por otra parte, "ateórica", aunque como nos recuerdan Álvarez, Esteban y Sauvagnat (16) se aprecia en su trasfondo la influencia del Departamento de Psiquiatría de la Universidad de Washington, los llamados "neokraepelinianos". Desaparece el concepto de neurosis y se limita el valor heurístico del de psicosis, se tiende a considerar las ciento cincuenta categorías propuestas como "válidas" y "fiables", culminando en una taxonomía "descriptiva" que pone en entredicho cualquier tipo de psicogénesis. El DSM-III, y sus sucesores a continuación, impusieron el paradigma médico en psicopatología, articulado con el behaviorismo por medio del empirismo, tan querido éste por uno y otro. En opinión de los autores citados, que compartimos aunque sea políticamente incorrecta o tal vez por ello, los DSM se revelan decididamente al servicio de los intereses económicos de la industria farmacéutica y de las compañías de seguros médicos, como se aprecia en la inflación de trastornos de ansiedad, afectivos y psicóticos, es decir, los correspondientes a los tres grandes grupos de psicofármacos. Esta clasificación, que se ha pretendido convertir en un manual de psicopatología cuando no es más que un catálogo de trastornos sin un principio organizador claro, ha tendido a asentar la vieja noción de entidad nosológica natural, aquélla que pretendía describir un proceso morboso o enfermedad según el modelo de la medicina interna: etiología, patogenia, anatomía patológica, sintomatología, curso y evolución (no hace falta recordar que en nuestra disciplina ni 
ORIGINALES Y REVISIONES

siquiera referente a las psicosis de base más firmemente considerada "biológica" como la esquizofrenia, tenemos claras las cuestiones de la etiología o la patogenia, más allá de posiciones de fe en espera de la llegada, siempre inminente, de la prueba definitiva). Como concluyen el abordaje de este tema Álvarez, Esteban y Sauvagnat, la enseñanza de la psiquiatría ha degenerado en una mera técnica y una huidiza práctica clínica de la atención de las enfermedades mentales, a las que se ha terminado por sustraer toda brizna de subjetividad.

Para ir cerrando este primer apartado de nuestro trabajo, nos gustaría detenernos en unas palabras de Fernando Colina (17), también muy críticas con el paradigma médico imperante y la visión de la psiquiatría que implica. Las citaremos casi textualmente, reconociendo nuestra incapacidad para saber expresar nuestras propias ideas, coincidentes en muchos puntos, con esa belleza y esa fuerza: "[...] en la actualidad [...] vivimos un idilio de la Psiquiatría con la Medicina de una intensidad casi desconocida. Aunque a cambio de estos amores, inducidos por intereses personales (identidad, resistencias subjetivas, pereza intelectual), sociales (prestigio, reconocimiento, ideología) o materiales (ganancias, presión comercial), la Psiquiatría se ha quedado sin espacio para respirar. [...] Una psiquiatría sólo biológica es como un hierro de madera que sostiene sin solidez, amputa la interpretación y nos obliga, sin justificación suficiente, a la falacia de naturalizar más los fenómenos anormales que los normales, como si la tristeza y la pereza, la pena y la desgana, ganaran rango fisiológico por el hecho de transformarse en depresión e inhibición, esto es, en mucha tristeza y mucha pereza, en mucha pena y mucha desgana. [...] Recordemos, por otra parte, que aquella actitud perturbadora y dogmática, que ya Jaspers denunciaba como "mitología cerebral", permanece activa en la grandilocuente "década del cerebro" o bajo los nuevos aires de la "mitología genética". Esta observación, sin embargo, no supone negar los avances de las neurociencias, pero se opone frontalmente a que una neurología conjetural anule la práctica clínica a la espera de un futuro prometedor. Lo peligroso de la mitología cerebral reside en que su expectativa de hallazgos, en general lejana, suspenda la interpretación psicopatológica excusándose en la tranquilidad de su verosimilitud y en la inminencia de una hipotética promesa".

Hasta aquí, la primera parte de nuestro trabajo, donde hemos querido señalar la, en nuestra opinión poco discutible, absoluta preponderancia del paradigma médico (término que, siguiendo a Luque y Villagrán, preferimos al de modelo médico) y, a través de él, el triunfo casi completo de la llamada psiquiatría biológica en nuestro entorno. Aunque, por supuesto, como cierta aldea gala, algunos valientes resisten la invasión y por ello hemos recogido sus palabras en las líneas precedentes. En mucho compartimos esas opiniones de Desviat, Álvarez o Colina, por ejemplo. Nos hemos detenido también en comentar someramente 
algunas pinceladas acerca de la (mal) llamada medicina basada en la evidencia, herramienta fundamental con aspiraciones de paradigma totalizador, de la citada psiquiatría biológica que nos rodea. Hoy en día, en nuestros campos profesionales, nos bombardean a diario con estudios propios de la MBE, brillantes ensayos clínicos, gloriosos metaanálisis, que muchas veces no hacen otra cosa que confirmar repetidamente ciertos correlatos biológicos en determinados trastornos mentales, así como el buen funcionamiento de nuestro arsenal terapéutico, por supuesto de eficacia y seguridad probadas (aunque luego ningún estudio nos explica por qué fármacos eficaces y seguros son dejados a un lado ante la aparición de otros menos probados sólo porque así lo sugieren representantes comerciales de la empresa que vende ese fármaco, con el apoyo de estudios patrocinados por esa misma empresa y habitualmente coincidiendo con los momentos inmediatamente posteriores a la caducidad de la patente del primer fármaco, ayer imprescindible y hoy obsoleto). Y sin embargo, contagiados tal vez de fervor positivista y espíritu científico, nos asaltó en un momento determinado una duda: ¿no estaremos dando por cierta sin pruebas suficientes nuestra hipótesis de que la práctica clínica de la psiquiatría en nuestro entorno está obsesionada por seguir los pasos marcados por la MBE, dentro del paradigma médico imperante? Es decir, opinamos que la influencia de la MBE y su afán cientifista en nuestra práctica clínica es exagerada y posiblemente nos ciega ante otros aspectos que creemos propios e ineludibles de nuestra disciplina, del orden de la escucha, la narración, la interpretación... para llegar al sujeto que sufre, más allá de los síntomas que gritan. Pero nuestra opinión no está probada. ¿Realmente la MBE tiene esa influencia que creemos en la práctica clínica psiquiátrica en nuestro entorno? Nos proponemos ahora comentar unos cuantos estudios, que nos han parecido interesantes, para examinar sus resultados y la aplicación de los mismos a nuestra práctica habitual.

Donde se da cuenta de diferentes estudios (científicos, eso sí) con resultados un tanto llamativos.

En 2005, Fergusson y colaboradores publican en el British Medical Journal (18) un trabajo titulado: "Asociación entre intentos suicidas e inhibidores selectivos de la recaptación de serotonina: revisión sistemática de ensayos clínicos aleatorizados". Esta revisión incluyó un total de 87.650 pacientes y detectó un incremento significativo en la tasa de intentos suicidas en los pacientes que recibieron ISRS comparado con el grupo placebo. También se puso de manifiesto un incremento en la tasa de intentos suicidas para ISRS en comparación con otros 
ORIGINALES Y REVISIONES

agentes terapéuticos, excepto los antidepresivos tricíclicos, con los que no hubo diferencia. La conclusión de los autores fue que se había documentado una asociación entre los intentos de suicidio y el uso de ISRS.

En febrero de 2008 se publica en la revista PLoS Medicine un metaanálisis (19) para estudiar la relación entre la severidad inicial de la depresión y la eficacia de la medicación antidepresiva, a partir de datos suministrados por la FDA americana (Food and Drug Administration). Se solicitó y recibió de la FDA los ensayos clínicos correspondientes a los estudios facilitados por la industria farmacéutica para conseguir la aprobación de la indicación antidepresiva de fluoxetina, venlafaxina, nefazodona y paroxetina. Los datos procedían tanto de estudios publicados como no publicados.

El metaanálisis incluyó cinco ensayos clínicos con fluoxetina, seis con venlafaxina, ocho con nefazodona y dieciséis con paroxetina, lo que supuso un total de 5.133 pacientes, de los que 3.292 fueron asignados aleatoriamente a recibir medicación y 1.841 asignados aleatoriamente a placebo. Los autores, analizados los datos, concluyen que no hay diferencia estadísticamente significativa en la respuesta antidepresiva entre los grupos con placebo y los grupos con cualquiera de los cuatro antidepresivos estudiados. Esto no significa que no se obtenga respuesta alguna. Al contrario, ambos grupos de pacientes deprimidos mejoran, pero sin diferencia significativa entre el principio activo y el placebo, excepto en los casos más severos de las depresiones graves, donde sí aparece un efecto estadísticamente significativo que coloca a los antidepresivos ligeramente por delante.

Los autores del metaanálisis concluyen que, dados estos datos, parece haber pocos indicios que apoyen la prescripción de esta medicación antidepresiva, excepto a los paciente más gravemente deprimidos, a menos que los tratamientos alternativos ya hayan sido probados sin resultado. Se menciona expresamente el empleo de la psicoterapia.

Este estudio, que además cumple el "patrón oro" de ser un metaanálisis de ensayos clínicos aleatorizados, es claro en sus conclusiones. Sin embargo, este reciente ejemplo de aplicación de la MBE no parece haber supuesto ningún cambio en los hábitos de prescripción en nuestro entorno. Es cierto que muchas veces los estudios pueden pasar desapercibidos entre la gran cantidad de bibliografía que se publica hoy en día. Además, estos estudios también tardan en ser incluidos, cuando lo son, en los tratados más conocidos de la especialidad, lo que a su vez puede influir en que los profesionales tardemos en tener acceso a esta información y, por lo tanto, no pueda aplicarse a la práctica cotidiana. Sin embargo, en este caso es una excusa poco creíble: los resultados de este metaanálisis fueron publicados en al menos dos diarios de tirada nacional en nuestro país ( $E l$ País y El Mundo de 26 de febrero de 2008) pero, a pesar de ello, al parecer los seguimos recetando sin gran pudor para cualquier tipo de depresión (o trastorno 
ansioso-depresivo, o distimia, o trastorno adaptativo, o como los queramos llamar...), sin preocuparnos en exceso de que su eficacia no esté nada clara mientras que sus efectos secundarios son bastante evidentes: molestias gastro-intestinales, mareos, hipertensión, extrapiramidalismo, ansiedad, disfunción sexual, síndrome serotoninérgico... No olvidemos tampoco que algunos de estos antidepresivos cuya eficacia ha sido cuestionada han servido a su vez de punto de comparación para demostrar la utilidad de otros de comercialización posterior, con la confianza de ser al menos tan eficaces como... Si un nuevo antidepresivo ha demostrado en un ensayo clínico ser al menos tan eficaz como uno de estos cuatro, que a su vez ha demostrado ser igual de eficaz que el placebo, ¿dónde deja eso la confianza en ese nuevo fármaco?, ¿dónde debería haber quedado ya nuestra confianza en el antiguo?, ¿no somos tan científicos y seguidores de la MBE?, ¿por qué no influyen pues en nuestra práctica habitual este tipo de estudios?

En la revista Schizophrenia Bulletin, poco sospechosa de intereses psicoanalíticos o amagos antipsiquiátricos, se ha publicado recientemente un artículo proponiendo un replanteamiento en la prescripción de antipsicóticos (20). Los autores llevan a cabo una revisión de la literatura reciente en lo referente a estos fármacos. Siguiendo dicho trabajo, vemos que en la revista JAMA en 2003 (21) se publicó un ensayo clínico aleatorizado y doble ciego con 309 pacientes, a doce meses, comparando olanzapina y haloperidol en cuanto a coste-efectividad. La dosis de olanzapina osciló entre 5 y $20 \mathrm{mg}$ /día, y la de haloperidol, entre 5 y 20 $\mathrm{mg}$ /día. Como los mismos autores comentan, los resultados fueron inesperados: no se observaron diferencias en la eficacia ni en la mayoría de efectos secundarios entre olanzapina y haloperidol en pacientes con diagnóstico de esquizofrenia. Hay que tener en cuenta que el haloperidol, al igual que la olanzapina, se administró con anticolinérgicos profilácticos. La conclusión del estudio fue que la olanzapina no demostraba ventajas comparada con el haloperidol (en combinación con los mencionados anticolinérgicos profilácticos) en cumplimiento, síntomas de esquizofrenia, síntomas extrapiramidales o calidad de vida, y sus beneficios en menor acatisia y mejora de la cognición debían ser equilibrados con los problemas de aumento de peso y mayor coste.

El estudio CATIE $(22,23)$, por su parte, fue un ensayo clínico aleatorizado y doble ciego, que incluyó 1.493 pacientes con diagnóstico de esquizofrenia. Los pacientes se distribuyeron en diferentes grupos: olanzapina (de 7,5 a $30 \mathrm{mg} /$ día), perfenazina (de 8 a $32 \mathrm{mg} /$ día), quetiapina (de 200 a $800 \mathrm{mg} /$ día), risperidona (de 1,5 a $6 \mathrm{mg} /$ día) y ziprasidona (de 40 a $160 \mathrm{mg} /$ día) y fueron seguidos 18 meses. Los resultados mostraron que el $74 \%$ de los pacientes abandonaron la medicación antes de los 18 meses (64\% para olanzapina, $75 \%$ para perfenazina, $82 \%$ para quetiapina, $74 \%$ para risperidona y $79 \%$ para ziprasidona). El tiempo transcurrido hasta el abandono de la medicación fue, de forma estadísticamente 
ORIGINALES Y REVISIONES

significativa, mayor para olanzapina que para risperidona o quetiapina, pero no hubo diferencia estadísticamente significativa con los grupos de perfenazina o ziprasidona. El tiempo transcurrido hasta el abandono por efectos secundarios fue similar entre los grupos, pero mientras que en el de olanzapina se produjeron más abandonos por aumento de peso o efectos metabólicos, en el de perfenazina hubo más por efectos extrapiramidales. El estudio CATIE no demostró ventajas significativas en ninguno de los cuatro antipsicóticos atípicos en comparación con la perfenazina en cuanto a las medidas de síntomas, efectos neurológicos secundarios, calidad de vida, empleo o función neuropsicológica. Como señalan Rosenheck y colaboradores, el estudio CATIE ha recibido distintas críticas en cuanto a supuestas limitaciones metodológicas, pero que serían en su mayoría igualmente aplicables a los estudios previos que mostraban ventajas de los antipsicóticos atípicos frente a los clásicos. Este autor plantea que el uso de dosis de moderadas a elevadas de haloperidol sin anticolinérgicos profilácticos en esos estudios anteriores puede haber proporcionado una ventaja improcedente a los antipsicóticos atípicos.

El estudio CUtLASS (24) fue un ensayo clínico multicéntrico aleatorizado que se llevó a cabo en el Reino Unido, bajo el patrocinio del gobierno. Partiendo de la percepción existente de que los antipsicóticos atípicos son más efectivos, tienen menos efectos adversos y son preferibles para los pacientes antes que los clásicos, se propuso comprobar la hipótesis de que los citados atípicos se asociarían con mejora en la calidad de vida en comparación con los antipsicóticos clásicos. El estudio tuvo una duración aproximada de un año, con medidas en las semanas 12, 26 y 56. Participaron 227 pacientes con diagnóstico de esquizofrenia y trastornos relacionados, que fueron valorados para revisión de medicación a causa de respuesta inadecuada o efectos adversos a las previas. La prescripción fue aleatorizada entre antipsicóticos típicos y atípicos, con determinación del fármaco concreto en cada grupo hecha por elección del psiquiatra. Se emplearon, entre otras, escalas de medida de calidad de vida, síntomas, efectos adversos y satisfacción del paciente.

Los resultados demostraron que la hipótesis de que mejoraría la calidad de vida, a lo largo del año de estudio, en los pacientes con antipsicóticos atípicos quedaba descartada. Los pacientes del grupo de antipsicóticos clásicos mostraron una tendencia, no estadísticamente significativa, hacia mayor mejoría en las escalas de calidad de vida y de síntomas. Los participantes no informaron preferencias claras por ninguno de los grupos. El estudio tampoco observó ventajas de los antipsicóticos atípicos frente a los clásicos en cuanto a efectos secundarios.

Parece clara la percepción, si nos molestamos en fijarnos en estudios como los comentados previamente, de que los estudios gubernamentales independientes cuestionan las conclusiones de la investigación anterior que, en gran medi- 
da, estaba patrocinada por la industria. Continuando la revisión bibliográfica de Rosenheck y colaboradores, merece la pena citar también una revisión Cochrane de estudios de primer episodio psicótico (25) en la que se ha concluido: "no está claro si el uso de antipsicóticos de nueva generación realmente hace que el tratamiento procure menos molestias y mejore el cumplimiento a largo plazo".

Finalmente, tras reseñar estos y otros estudios, Rosenheck y colaboradores, en su artículo en el Schizophrenia Bulletin, recomiendan como tratamientos de primera elección para la esquizofrenia la risperidona (teniendo en cuenta que es el antipsicótico atípico más barato y cuenta con presentación genérica) y los antipsicóticos de primera generación de potencia intermedia, citando expresamente la perfenazina. Como comentan los autores del citado artículo, el gasto anual en antipsicóticos atípicos en EEUU llegó en 2005 a 11.600 millones de dólares. Recogemos textualmente unas líneas especialmente lúcidas, aun cuando su obviedad debería tal vez sonrojarnos: "La priorización de tratamientos de coste elevado como los antipsicóticos de segunda generación sólo es racional si procuran resultados de salud superiores en comparación con otras medicaciones en una medida que justifique su coste adicional. [...] los antipsicóticos de segunda generación no son más eficaces que los antipsicóticos de primera generación, pero sí considerablemente más caros, y las diferencias entre efectos secundarios son complejas, varían entre los fármacos individuales y no favorecen ninguna de las clases. En nuestra opinión, las diferencias en los costes merecen una especial atención, ya que son claras, fiables y considerables, mientras que las diferencias en la eficacia son limitadas y los indicios de diferencias en los efectos secundarios [...] parecen más variables y pueden compensarse entre sí en cuanto al efecto neto. No conocemos ningún contraargumento de que los antipsicóticos de segunda generación merezcan su coste anual de 11.600 millones de dólares ni ninguna justificación específica para el uso ineficaz de recursos en el caso de estos medicamentos en particular". Poco se podría añadir a este párrafo y, desde luego, nada que lo mejorara.

\section{A modo de conclusiones.}

Tras comentar estos estudios, tenemos forzosamente que concluir que nuestra hipótesis ha quedado muy debilitada. Partíamos de la base de que la psiquiatría de orientación biológica, situada en los parámetros del paradigma médico y esencialmente empirista y positivista, domina el escenario y que, en su quehacer científico y exclusivamente científico, emplea como herramienta básica la medicina basada en la evidencia para obtener sus conclusiones y decidir sus actua- 
ORIGINALES Y REVISIONES

ciones. Pero, sin embargo, nos encontramos ahora con una serie de metaanálisis de ensayos clínicos aleatorizados, publicados en revistas científicas conocidas y de prestigio, incluso algunos reseñados por medios de comunicación populares de amplia distribución, como comentamos previamente, cuyos resultados no parecen haber afectado la práctica clínica psiquiátrica, al menos no en nada que hayamos podido percibir en nuestro entorno.

Nos hemos encontrado con estudios que no han demostrado beneficio superior al del placebo para algunos de los más empleados antidepresivos, modelos de comparación a su vez de otros posteriores, así como estudios que encuentran una asociación estadísticamente significativa entre ISRS e intentos suicidas, sin que ni una información ni la otra parezcan haber afectado para nada la prescripción habitual de psiquiatras y médicos en general. Nos llegan incluso en ocasiones pacientes preguntando por tales informaciones aparecidas en la prensa generalista, actitud que suele encajarse con cierto fastidio y restando valor a los mencionados trabajos, que probablemente no han sido leídos por la mayoría de los profesionales, a pesar de ser de acceso fácil, completo y gratuito en la red. Parece que no queremos ni considerar la posibilidad de emplear métodos psicoterapéuticos como alternativa al tratamiento exclusivamente farmacológico.

No hemos querido comentar una serie de trabajos (26) que comparan, en primeros episodios de psicosis, la efectividad de abordajes psicoterapéuticos con dosis bajas y durante muy poco tiempo de antipsicóticos, o bien sin ninguna medicación en absoluto, con abordajes estándar con neurolépticos porque, a pesar de sus resultados francamente llamativos, serían fácil objeto de críticas bajo acusaciones de "antipsiquiatría", y hemos preferido centrarnos en estudios impecables desde el punto de vista "científico". Siguiendo esta idea, comentamos varios metaanálisis de ensayos clínicos aleatorizados que cuestionan abiertamente la supuesta mayor eficacia y mejor tolerabilidad de los antispicóticos atípicos frente a los neurolépticos clásicos. La cuestión en términos de coste para el sistema público en el que trabajamos (y, como no deberíamos olvidar, para el que trabajamos) es sencillamente escandalosa. Si tales metaanálisis son tomados en consideración (y no se nos ocurre razón científica para que no lo sean), no parece haber motivo para seguir con las actuales tendencias de prescripción, que inician el tratamiento con un antipsicótico atípico, en muchos casos llegando a dosis por encima de ficha técnica y en otros muchos combinándolo con otros atípicos, disparando el gasto sin gran preocupación o especial pudor. Tomando como ejemplo el estudio que comparaba olanzapina con haloperidol, si nos molestáramos en realizar un cálculo simple obtendríamos que un tratamiento con olanzapina a 20 mg/día costaría unos 3600 euros al año, mientras que con haloperidol a $20 \mathrm{mg}$ / día (ambas las dosis máximas empleadas en el citado estudio), el coste sería de 84 euros. Teniendo en cuenta que en la revisión no se encontró diferencia estadísti- 
camente significativa para eficacia o tolerabilidad, la sola duda de cuál prescribir sería, dentro de los parámetros científicos de la MBE, sencillamente absurda. Y no olvidemos que el dinero para pagar los psicofármacos que recetamos es el mismo que debe servir para hacer funcionar el sistema público de salud (que, por otra parte, nos paga el sueldo). En una época de crisis, donde ya se comenta la posibilidad de seleccionar qué pacientes con cáncer pueden recibir los tratamientos quimioterápicos más costosos en términos de beneficio esperable, se convierte en una obligación ética el cuidar nuestra prescripción a la hora de administrar un dinero que es público, limitado y escaso.

Así pues, si no es la MBE la que marca la línea directriz de la psiquiatría biológica, y por lo hablado hasta ahora pensamos que no es así, ¿cuál es esa línea directriz y quién la marca? La respuesta está, por supuesto, sujeta a opiniones, pero la nuestra creemos que la hemos expresado claramente: la industria farmacéutica domina férreamente nuestra práctica clínica cotidiana. Estudios metodológicamente inadecuados, con tamaños muestrales no representativos y recogidas de datos dudosas, son tomados como si fueran verdades innegables a la hora de recomendar la prescripción de diferentes fármacos (que nos demuestran supuestamente eficacias maravillosas a dosis que, pocos meses después, el propio laboratorio nos dirá que son muy bajas y que es necesario duplicar o triplicar... ¿ y no nos preguntamos cómo aquéllos primeros estudios pudieron obtener resultados tan positivos con dosis que ahora son inadecuadamente bajas?). Metaanálisis como los reseñados en este trabajo son ignorados sistemáticamente.

No es la MBE la que es cuestionada por la visión biologicista imperante, por supuesto, sino sólo los resultados que van en contra de los intereses mercantiles de la industria que marca nuestra agenda de eventos científicos, nuestra formación continuada, la docencia de nuestros residentes y, lo que es más preocupante, la prescripción que indicamos a los pacientes que confían en nosotros y pagamos con el dinero público que se nos ha encargado administrar. Nos tememos que el Zeitgeist psiquiátrico en el que estamos insertos no es sólo biologicista sino más bien, y lo decimos con tristeza, esencialmente comercial $(27,28)$.

De todas maneras, aunque no hubo suficientes justos para que Dios perdonara Sodoma y Gomorra, la posición dominante de la psiquiatría biologicista es mayoritaria pero no hegemónica. Hay otras visiones (29), otras formas de entender el paciente, la enfermedad, el malestar. Hay abordajes psicoterapéuticos que pueden aprenderse, enseñarse y aplicarse. Hay posibilidad de emplear la farmacoterapia a nuestra disposición con racionalidad, sin excesos, buscando beneficios pero sin ignorar efectos secundarios o costes. Hay otra psiquiatría, que existe y debe promocionarse, si queremos salvar la dignidad de nuestra disciplina y la nuestra propia. Desde otros paradigmas se dan otras formas de ver la psiquiatría pero, incluso desde el mismo paradigma médico, hay otra forma de 
ORIGINALES Y REVISIONES

hacer psiquiatría más allá del control de la industria farmacéutica que intentamos denunciar aquí.

Stephen J. Bergman, bajo el seudónimo de Samuel Shem, escribió hace ya unos años el libro "Monte Miseria", donde contaba las peripecias de un residente de psiquiatría en su primer año de formación. En tono de tragicomedia, el autor nos narraba toda suerte de maldades, abusos y negligencias que constituían la norma y no la excepción de la práctica psiquiátrica. Uno de nosotros escribió, en relación a esta obra, un humilde análisis de título pretencioso: De traidores, víctimas y deserciones: Diario de guerra en Monte Miseria (30) y querríamos rescatar sus palabras finales para poner también aquí nuestro final: "Si la psiquiatría [...] está enferma, sucia y violada, nuestro deber es hacerla levantarse, recuperar la dignidad y ponerla a trabajar en sus asuntos, que son los nuestros. No vale mirar para otro lado ni huir a las montañas". 


\section{BIBLIOGRAFÍA:}

(1) Vallejo Ruiloba, J. Introducción a la psicopatología y la psiquiatría $5^{\circ}$ ed., Barcelona, Masson, 2002.

(2) Klerman, G., "The evolution of a scientific nosology", en Shershow, J. (dir.), Schizophrenia: science and practice. Cambridge, Harvard University, 1978.

(3) Luque, R. y Villagrán, J.M., Psicopatología descriptiva: nuevas tendencias, Madrid, Editorial Trotta, 2000.

(4) BerRIOs, G.E., "Psicopatología descriptiva: aspectos cuantitativos y cualitativos", en Cervera, S. y Martínez-Vila, E. (eds.), Nuevas perspectivas en psiquiatría biológica, Pamplona, Eurograf, 1995.

(5) MAHER, B., "Modelos para el estudio de la psicopatología", en Maher, B., Introducción a la investigación en psicopatología, Madrid, Taller de Ediciones, 1974.

(6) LÁZARO, J., "De la evidencia ilusoria a la incertidumbre razonable: Introducción histórica", Archivos de psiquiatría, 2000, supl.3, pp. 5-26.

(7) Navarro, F.A., Diccionario crítico de dudas inglés-español de medicina, Madrid, McGraw-Hill Interamericana, 2000.

(8) Desviat, M. "Psiquiatría y evidencia. Los límites de la función del clínico", en Baca, E. y Lázaro, J. (eds.), Hechos y valores en psiquiatría, Madrid, Ed. Triacastela, 2003.

(9) Kunn, T.S., La estructura de las revoluciones científicas, Mexico, FCE, 1975.

(10) Laupacis, A.; Sackett, D.L.; Roberts, R.S., "An assessment of clinically useful measures of the consequences of treatment", New England Journal of Medicine, 1988, 318, pp. 1.728-33.

(11) Evidence-based medicine working group, "A new approach to teaching the practice of medicine", JAMA, 1992, 268, pp. 2.420-5.

(12) SACKetT, D.L. y otros, "Evidence based medicine: what it is and what it isn't", British Medical Journal, 1996, 312, pp. 71-2.

(13) Roca Bennasar, M. y Cañellas Dols, F., "Psiquiatría basada en pruebas", en Vallejo Ruiloba, J. y Leal Cercós, C., Tratado de psiquiatría, Barcelona, Ars Medica, 2005.

(14) Detre, T., "The future of psychiatry", American Journal of Psychiatry, 1987, 144, 5, pp. 621-5.

(15) GuzE, S.B., "The future of psychiatry and the medical model", Journal of nervous and mental diseases, 1993, 181, 10, pp. 593-4.

(16) Állvarez, J.M.; Esteban, R.; Sauvagnat, F., Fundamentos de psicopatología psicoanalítica, Madrid, Ed. Síntesis, 2004.

(17) Colina, F., “Actualidad hermenéutica de las psicosis", Frenia. Revista de historia de la psiquiatría, 2002, vol. II, fascículo 2, pp. 109-119.

(18) Fergusson, D. y otros, "Association between suicide attempts and selective serotonin reuptake inhibitors: systematic review of randomised controlled trials", British Medical Journal 2005, 330, pp. 396.

(19) KIRSCH, I. Y OTROs, "Initial severity and antidepressant benefits: a meta-analysis of data submitted to the Food and Drug Administration", PLoS Med, 2008, 5, 2, e45 doi: 10.1371/journal.pmed. 0050045 .

(20) RosenheCK, R.A. y OTROs, "Replanteamiento de la política de prescripción de antipsicóticos”, Schizophrenia Bulletin (Ed Esp), 2009, 4, 1, pp. 58-63.

(21) RosenheCK, R.A. Y otros, for the Department of Veterans Affairs Cooperative Study Group on the Cost-Effectiveness of Olanzapine, "Effectiveness and cost of olanzapine and haloperidol in the treatment of schizophrenia", JAMA, 2003, 290, pp. 2693-2702.

(22) LIEBERMAn, J.A. Y OTRos, for the CATIE Investigators. "Effectiveness of antipsychotic 
drugs in patients with chronic schizophrenia: primary efficacy and safety outcomes of the Clinical Antipsychotic Trials of Intervention Effectiveness (CATIE) Schizophrenia Trial", N Engl J Med, 2005, 353, pp. 1209-1223.

(23) SWARTZ, M. Y OTROS, "Effects of antipsychotic medications on psychosocial functioning in patients with chronic schizophrenia: findings from the NIMH CATIE Study", Am J Psychiatry, 2007, 164, pp. 428-436.

(24) JoNES P.B. y OtRos, "Randomized controlled trial of effect on quality of life of second generation versus first generation antipsychotic drugs in schizophrenia - CUtLASS1", Arch Gen Psychiatry, 2006, 63, pp. 1079-1087.

(25) Rummel, C. у отRos, "New Generation Antipsychotics for First Episode Schizophrenia", Cochrane Database of Systematic Reviews: Reviews 2003 Issue 4 John Wiley \& Sons, Ltd Chichester, UK Available at: http://www.mrw.interscience.wiley.com/cochrane/clsysrev/articles/CD004410/ frame. html. Accessed June 7, 2007.

(26) Mosher, L.R., "Intervención en un primer episodio de psicosis sin hospitalización ni fármacos", en Read, J., Mosher, L.R., Bentall, R.P. (eds.) Modelos de locura, Barcelona, Herder, 2006.

(27) González Pardo, H. y Pérez Álvarez, M., La invención de trastornos mentales, Madrid, Alianza Editorial, 2007.

(28) Mata Ruzz, I.; Ortiz Lobo, A., "Industria farmacéutica y psiquiatría", Revista de la Asociación Española de Neuropsiquiatría, 2003, 86, pp. 49-71.

(29) Read, J.; Mosher, L.R.; Bentall, R.P., Modelos de locura, Barcelona, Herder, 2006.

(30) García-Valdecasas Campelo, J., "De traidores, víctimas y deserciones: Diario de guerra en Monte Miseria", en Seminarios de la Unidad de Docencia y Psicoterapia Volumen 10, Mente y Literatura, Granada, Fundación Virgen de las Nieves, 2004.

\footnotetext{
* Jose García-Valdecasas Campelo. Psiquiatra. Hospital Universitario de Canarias. Servicio Canario de Salud. Amaia Vispe Astola. Enfermera Especialista en Salud Mental. Cristina Tobías Imbernón. P.I.R. Psicología Clínica $3^{\circ}$. Hospital Universitario de Canarias. Servicio Canario de Salud. Miguel Hernández González. M.I.R. Psiquiatría 3․ Hospital Universitario de Canarias. Servicio Canario de Salud.
}

\section{Correspondencia:}

Jose García-Valdecasas Campelo. Avenida de Madrid n ${ }^{\circ} 11$, portal 5, $8^{\circ}$ B. 38007. Santa Cruz de Tenerife. Islas Canarias. España. Correo electrónico: jose_valdecasas@hotmail.com.

** Recibido: 9/03/2009 